Copyright $@ 2010$ Institute of Electrical and electronics Engineers, Inc.

All Rights reserved.

Personal use of this material, including one hard copy reproduction, is permitted.

Permission to reprint, republish and/or distribute this material in whole or in part for any other purposes must be obtained from the IEEE.

For information on obtaining permission, send an e-mail message to stds-igr@ieee.org.

By choosing to view this document, you agree to all provisions of the copyright laws protecting it.

Individual documents posted on this site may carry slightly different copyright restrictions.

For specific document information, check the copyright notice at the beginning of each document. 


\title{
Estimation of Power Factor by the Analysis of Power Quality Data for Voltage Unbalance
}

\author{
Zahir J. Paracha ${ }^{1}$, Akhtar Kalam ${ }^{1}$, Ahmed M Mehdi ${ }^{1}$, M.T.O. Amanullah ${ }^{2}$ \\ ${ }^{1}$ School of Engineering and Science, Victoria University, Melbourne, Australia. \\ ${ }^{2}$ Faculty of Sciences, Engineering and Health, Central Queensland University, Rockhampton, Australia. \\ \{zahir.paraha, akhtar.kalam\}@vu.edu.au, ahmed.mehdi@research.vu.edu.aua.maungthanoo@cqu.edu.au
}

\begin{abstract}
Power Quality (PQ) has been identified as a complex and diversified problem across the board in electrical power industry. It has not only confused the power utilities but also has attracted the attention of its customers and equipment manufacturers. Although extensive research work is being done in this field but one of the main problem encountered by its stake holders is the voltage unbalance in electrical power system. The problem of voltage unbalance has affected the safety, reliability and economic efficiency at all levels in power industry. In this research Computational Intelligence Techniques have been used for efficiently predicting the power factor of unbalanced load of a power distribution system. The Principal Component Analysis Technique was used to find the optimized number of new dimensions of PQ data. Finally Feed Forward Back Propagation (FFBP) algorithm was used to estimate the power factor of a distribution network by analyzing the real power system parameters. This research highlights the importance of maintaining power factor close to unity for power utilities to achieve sustainable availability of quality supply of electrical power for its customers. The outcomes of the proposed techniques were compared and tested with the field results of a power utility in Victoria, Australia.
\end{abstract}

Index--Terms-Power Quality, Voltage Unbalance, Unbalanced loads, Principal Component Analysis.

\section{INTRODUCTION}

In electrical power system the management of voltage unbalance is of prime importance for provision of quality supply of electric power. Voltage unbalance is not restricted to only technical problems in a power utility but it is also concerned with economics for both power utility and its customers. The voltage unbalance contributes to poor power factor in electrical power system. Power companies aim to maintain power factor of the power system close to unity. However with the increase of nonlinear loads, it becomes difficult practically to eliminate the voltage unbalance in the distribution network [1]. There are power companies which impose a penalty to its customers if their load contributes towards poor power factor and voltage unbalance in electrical distribution networks. The close monitoring of power factor calculations by power companies forces the industrial customers to take all adequate steps to maintain their power factor close to unity. There may be one or more reasons for the voltage unbalance at one time in a power distribution network.
The voltage unbalance can be due to the changes in the voltage values at different phases.. The deviation phase angle also causes the voltage unbalance. According to IEEE definition [2], the voltage unbalance can be expressed by equation (1).

$$
\% v=\frac{\max (\overline{\nabla v})}{\bar{v}} .100 \ldots \ldots \ldots
$$

where $\% v$ is the percentage unbalance, $\overline{\nabla v}$ is the deviation in the average phase voltage and $\bar{v}$ is the average phase voltage of a distribution system.

The power factor (pf) is a relation between the actual power and the apparent power given by equation (2):

$$
p \cdot f=\cos \phi \text {. }
$$

Equation (3) stands for a balanced system whereas in case of unbalanced system, the power factor can be expressed by considering the harmonics power components of the current [3] as shown in equation (3).

$$
p . f=\frac{V I \cos \phi_{1}+\sum V_{x} I_{x} \cos \phi_{x}}{V} \ldots \ldots \ldots \ldots \text { (3) }
$$

In equation (3) $V I \cos \phi_{1}$ is related to fundamental frequency whereas the second term, $\sum V_{x} I_{x} \cos \phi_{x}$ relates to harmonics and voltage unbalances. This makes the estimation of power factor a difficult task as the harmonic components identification can not be calculated completely.

Different researcher worked on the estimation of different parameters of a power system. Kingsley et al [4] proposed an intelligent system for the estimation of harmonics originating from a single phase load but this system could not responded good for a three phase system. A neural network based system for power factor correction was proposed by Malik et al [5]. The concept of an intelligent power system was well published by Jim McDonald [6]. In this concept, some intelligent power electronics sensors were developed for efficient performance of a power distribution network.

Neural Networks (NN) have been proved to produce appreciable results and have the capability to accurately model the system. The neural network has great ability to deal with random alteration of different values of PQ data. The real PQ data used in this research is for voltage unbalance of the power 
distribution network in Victoria Australia.

In this research, the power factor is estimated by using computational intelligence techniques. The optimized power quality data parameters of voltage unbalances have been analysed for estimation of power factor for enhanced system performance. This paper is organised as follows:

Section II highlights the methodology used for analysing the voltage unbalances. The experimental results and discussion are written in Section III while conclusions are drawn in Section IV.

\section{MethodOLOGY FOR ANALYISNG VOLTAGE UNBALANCE}

In this research, intelligent algorithms have been used to analyze the power quality parameters of $66 / 22 \mathrm{KV}$ zone substation in Victoria, Australia. The PQ instruments at the substation monitor the voltage at the $22 \mathrm{kV}$ bus. The voltages are measured phase-to-phase. The voltage unbalance parameters have been recorded for period of nearly three months along with power factor of the power network. The following techniques are employed.

\section{A. Principal Component Analysis Techniques (PCAT)}

Due to larger number of attributes for monitoring the power quality data containing different parameters, it is very difficult to process all attributes. In many practical applications, principal component analysis technique (PCAT) is employed for finding those dimensions of power quality data where the spread of data is found to be maximum. In this PCAT the zero axis of the power quality data is shifted to a new axis depending upon the mean values of all data attributes. The covariances between all attributes are calculated to find the Eigen Vector [7]. Each Eigen Vector corresponds to new dimension of a Power Quality data which helps in better estimation of power factor.

\section{B. Feed Forward Back Propagation Algorithm (FFBP)}

After reducing the attributes of power quality data, Feed Forward Back Propagation (FFBP) is used to estimate the power factor of the system. Due to non convergence of a Multilayer Perceptron Algorithms (MLP), FFBP algorithm is proposed. In this Algorithm supervised techniques are employed. The Least Mean Squared technique [8] is applied by calculating the non linear error of the estimated values of power factor. The weight matrix of FFBP algorithm is calculated using gradient calculation method as shown by equation (4).

$$
\Delta w_{i k}^{m}=-\frac{\ell \partial E_{x}}{\partial \Delta w_{i k}{ }^{m}} \ldots \ldots \ldots \ldots . .(4)
$$

where $\Delta w_{i k}^{m}$ represents the weight from $i^{t h}$ to $k^{\text {th }}$ unit of $(m-1)^{\text {th }}$ neural network layer, $\ell$ is the learning rate of neural network and $E_{x}$ is the least mean square error which is calculated from the difference of current and desired power factor outputs of the neural network. The weight initialization is considered to be most effective approach in FFBP networks for better and efficient estimation of power factor.

\section{RESULTS AND DISCUSSION}

In this research NN techniques have been used to efficiently predict the power factor of the distribution system which is close to unity. The feed forward back propagation (FFBP) neural network has been found to have an inherited error tolerance for PQ real data used in this research. FFBP has proved to be the simplest method that can be employed for estimation of real PQ data with good predictable efficiency. The results of power quality data using Principal Component Analysis and Feed Forward Back propagation algorithm are discussed below.

\section{A. The Power Quality Data}

The Power Quality real data has been taken from a power distribution and management company in Victoria, Australia. It consists of fifteen attributes of voltage unbalance. Each attribute contains 2208 datasets. The raw average values of PQ voltage unbalance were used. The dataset consists of hourly average values of different parameter of voltage unbalance for consecutive ninety two days.

\section{B. Principal Component Analysis}

Fig.1. shows the plot of Eigen Values for reducing the attributes of the power quality data. Each Eigen Value corresponds to one new dimension of the real Power Quality data. Fig. 2. shows that total power quality data can be represented by two new dimensions without the loss of any data. (First Two Eigen Vectors)

\section{Loss of Data}

The Loss of Power Quality data has been shown in Fig. 3. It clearly indicates that $0 \%$ data is lost by considering two Principal components of Power Quality Data.

\section{Feed Forward Back Propagation Algorithm}

Ten percent of the data was reserved for testing purposes. On the average 93\% of accuracy was achieved. A FFBP Algorithm was implemented on a Neural Network. The Neural network consists of two layers as shown in Fig. 4. Tansigmoid function was used in the first Layer while Logsigmoid function was employed in the hidden Layer. The output Layer was the estimated Power factor at different parametric values of voltage unbalance of the power system network. The training error curve is shown in Fig. 5. The network achieved the convergence in 100 epochs. Table I shows the estimated and actual values of the power factor and their differences. The accuracy of estimated power factor helps in achieving the desired objectives of availability of quality supply of power to customers on sustainable basis. 


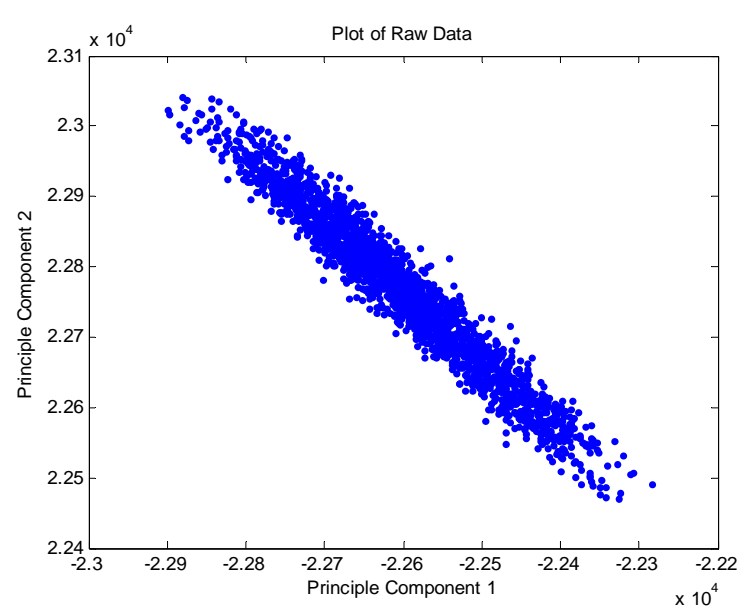

Fig. 2. Plot of PQ data in 2 Dimensions.

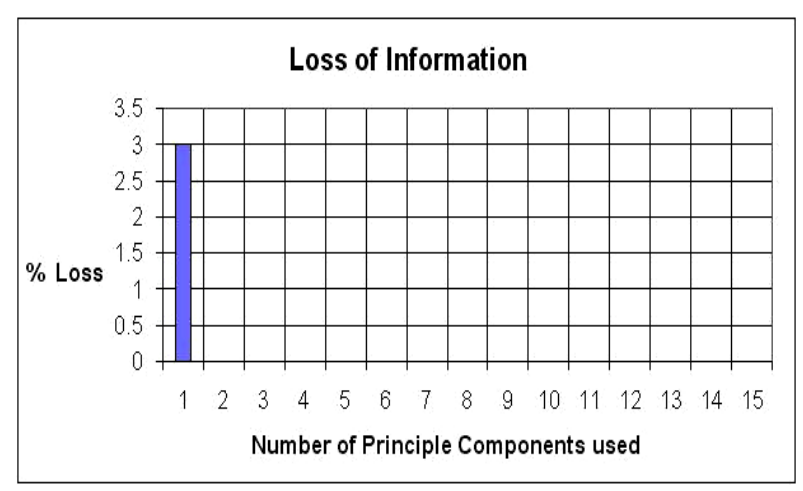

Fig. 3. Loss of Power Quality Data.

Table I

Predicted and Actual Values of Power Factor

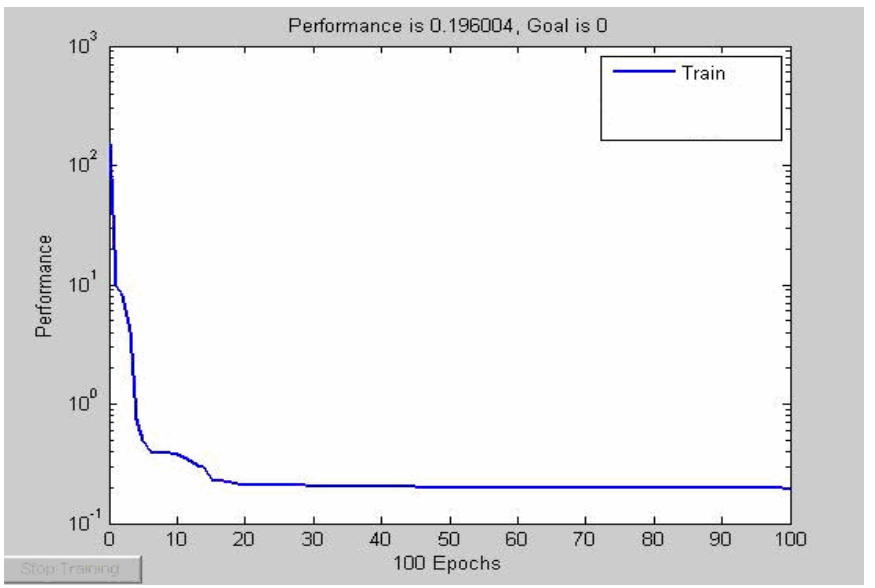

Fig. 5. Training Error Curve

\section{CONCLUSIONS}

\begin{tabular}{|c|c|c|c|}
\hline $\begin{array}{c}\text { Test } \\
\text { No. }\end{array}$ & $\begin{array}{c}\text { Predicted } \\
\text { Values of PF }\end{array}$ & $\begin{array}{c}\text { Actual Values } \\
\text { of PF }\end{array}$ & Difference \\
\hline 1 & 0.9240 & 0.9939 & 0.0699 \\
\hline 2 & 0.9262 & 0.9931 & 0.0669 \\
\hline 3 & 0.927 & 0.9925 & 0.0655 \\
\hline 4 & 0.9278 & 0.9911 & 0.0633 \\
\hline 5 & 0.9184 & 0.9811 & 0.0627 \\
\hline 6 & 0.9287 & 0.9712 & 0.0425 \\
\hline 7 & 0.9211 & 0.9965 & 0.0754 \\
\hline 8 & 0.9281 & 0.9983 & 0.0702 \\
\hline 9 & 0.92 & 0.9988 & 0.0788 \\
\hline 10 & 0.9292 & 0.9992 & 0.0700 \\
\hline
\end{tabular}

Power Quality (PQ) is a diversified issue and needs a lot of attention in computational analysis. This research clearly states that by employing efficient and advance intelligent techniques, power quality data can be monitored easily and system performance can be enhanced. In this research an appreciable accuracy of $93 \%$ for power factor estimation is achieved on voltage unbalances of a power quality data. The outcome of this research is that the proper estimation of power factor helps in safety, reliability and economical efficiency of the power system on long term basis.

\section{ACKNOWLEDGMENT}

The authors gratefully acknowledge Mr. Peter Wong, Manager Electricity Asset Management, Jemena Ltd. Australia for the help and support in this research.

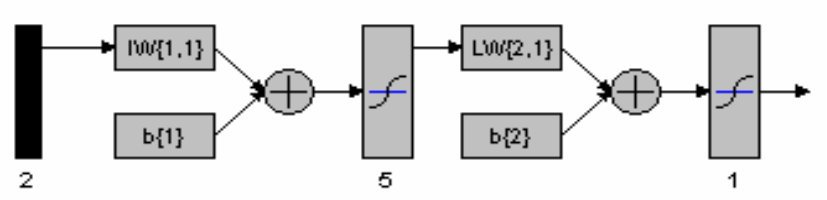

Fig. 4. Architecture of 2 Layer Feed Forward Back Propagation Neural Network.

\section{REFERENCES}

[1] Annette Von Jouanne, “Assessment of Voltage Unbalance”, IEEE Trans. on Power Delivery, Vol.16, No.4, October 2001, pp: 782-790.

[2] M. H. J. Bollen, "Definition of Voltage Unbalance", Journal, IEEE Power Engioneering Reviews, November 2002, pp: 49-50.

[3] F. P. Marafao, S. M. Declonann, and J.A.G. Marafao "Power Factor Analysis under Non-Sinusoidal and Unbalanced Systems" Proceedings of tenth international conference on Harmonics and Quality of Power, Vol.1, 2002, pp: 266-271.

[4] Kingsley Umeh and Azah Mohamed "Intelligent System for Identification of Harmonics Originating from Single Phase Nonlinear Loads”, Proc. of $19^{\text {th }}$ International South-East conference, 2005, pp: 137-142.

[5] Maiik E. Elbuluk Hei Wah Chan Iqbal Husain "Neural Network Controllers for Power Factor Correction of AC/DC Switching Converters”, Industry Applications Conference, $33^{\text {rd }}$ IAS Annual Meeting, 1998, pp: 1617-1624.

[6] Jim McDonald, "Adaptive intelligent power systems: Active distribution networks”, Journal, Elsevier Science, Energy Policy Vol. 36, pp: 43464351. 
[7] Lindsay I Smith, “A tutorial on Principal Component Analysis”, February 2002, pp: 1-26, Available online, [http://www.cs.otago.ac.nz], Last Accessed, January 1, 2009.

[8] Shafayat Abrar, Azzedine Zerguine and Maamar Bettayeb, "Recursive Least-Squares Backpropagation Algorithm for Stop and Go DecisionDirected Blind Equalization”, IEEE Trans. on Neural Network, Vol.13, No.6, November 2002, pp: 1472-1481. 